\section{Correr el riesgo: ¿desventaja social o capacidad?}

\section{Run the risk: social disadvantage or capability?}

Correr o risco: desvantagem social ou capacidade?
Luz Adriana Muñoz-Duque 1

doi: 10.1590/0102-311X00171617
Correspondencia

L. A. Muñoz-Duque

Universidad de Antioquia.

Calle 67, 53-108, bloque 9-409, Ciudad Universitaria, Medellín, Colombia.

luza.munoz@udea.edu.co

1 Universidad de Antioquia, Medellín, Colombia expresión de restricciones en las capacidades de los sujetos, partiendo de desventajas sociales que pueden estar normalizadas en su cotidianidad. Por otro lado, puede ser pensado como expresión de capacidad, en aquellos casos en los cuales es aceptado o admitido a través del ejercicio de la libertad, siempre que los sujetos que así se relacionan con el riesgo, lo conciban en consonancia con su idea de una vida buena, cuya construcción implica el desarrollo pleno de la capacidad de agencia. Finalmente, se presentan algunas reflexiones sobre la materia dentro del ámbito de la salud pública.

Asunción de Riesgos; Riesgo; Justicia Social; Libertad 


\section{Introducción}

Pese a tratarse de un término polisémico, el riesgo ha adquirido significados ligados a desenlaces negativos, al ser comúnmente entendido como peligro o consecuencia, como posible adversidad o amenaza 1,2. En la época actual, caracterizada por una ampliada sensación de inseguridad 3,4 y por una preocupación anticipatoria acentuada, algunas disciplinas se han interesado en la previsión de fenómenos y eventos, hacen uso del concepto de riesgo desde diferentes ópticas y construyen conocimiento adoptando perspectivas y dispositivos para hacer frente a la incertidumbre. En el ámbito de la salud, principalmente por la vía de la epidemiología, el concepto de riesgo ha tenido un desarrollo importante, con un privilegio de su acepción probabilística, referida al comportamiento de los eventos en salud al ser investigados respecto de asociaciones particulares. Así pensado, el riesgo tiene un componente anticipatorio y, al hacer posible la predicción temporal y espacial, otorga un carácter generalizador al conocimiento epidemiológico 5,6,7.

Algunos autores han planteado críticas a este abordaje, subrayando limitaciones como la dificultad para determinar asociaciones entre variables de tipo social y otras denominadas biológicas, pese a que, en muchos casos, parecieran claras las conexiones entre ellas 8 . También se ha señalado que esta aproximación concibe el riesgo como con una "existencia autónoma" y desconsidera los contextos socioculturales en los que se encuentran los sujetos 5 . Además, esta mirada tiene una pretensión prescriptiva, a partir de la cual se determinan pautas comportamentales orientadas a la prevención del riesgo y la promoción de estilos de vida saludables, asunto que permite responsabilizar a los sujetos por sus resultados en salud, mientras se genera un escenario propicio para el favorecimiento de una industria de la determinación, evaluación y transferencia de riesgos, para la aparición de una oferta de servicios, productos, especialistas, nuevas tecnologías y programas para la prevención de riesgos 1,5, cuya definición puede estar atada a intereses económicos, políticos o de otra índole 1,2, lo que supone cuestionamientos acerca de quién define lo riesgoso, cuáles son esos intereses que subyacen a esta definición y cuáles son los asuntos que, interesa, sean leídos por las personas como riesgosos o como prácticas y conductas de riesgo.

En un contexto en el que el riesgo ha sido asociado a "malas elecciones" en los estilos de vida de los sujetos y, consecuentemente, a irresponsabilidad o culpa, algunos autores han buscado comprender las bases sociales, así como las implicaciones éticas y políticas que subyacen a los comportamientos de riesgo, lo cual ha dado lugar a otros abordajes alternativos expresados, por ejemplo, en discusiones sobre la vulnerabilidad, esto es, sobre aquellos aspectos relacionados con una mayor susceptibilidad de los sujetos respecto de alteraciones en su salud, y con una disponibilidad menor de recursos para protegerla 9 .

En una vía afín, se ha planteado la relación entre situaciones de riesgo y asuntos asociados a inequidades sociales 10,11,12, sentido en el cual el riesgo no puede considerarse al margen de unas dinámicas particulares de justicia, en la medida en que puede derivar de situaciones de desventaja social de sujetos con pertenencias sociales específicas. Desde estas lógicas, se amplía la idea del riesgo como ligado a procesos sociales que lo condicionan.

El interés por pensar otras dimensiones del riesgo también ha dado lugar al abordaje de, verbigracia, la manera como los sujetos se relacionan con riesgos propios de sus contextos de vida, no necesariamente para rechazarlos y generar protecciones frente a ellos, sino, incluso, para asumirlos como parte de su cotidianidad 2,12,13, miradas estas que están lejos de desestimar procesos sociales que configuran tanto la definición de los riesgos como la respuesta de los sujetos frente a estos.

Con atención a estos planteamientos, el artículo se orienta a reflexionar alrededor de una lectura del riesgo desde una perspectiva de justicia social, con fundamento en ideas propias del enfoque de las capacidades, particularmente, en su comprensión de justicia, libertad, agencia y en el concepto de preferencias adaptativas, al considerar la posibilidad de naturalización tanto de la falta de opciones para elegir, como de la exposición misma a ciertos riesgos. En virtud de ello, las siguientes líneas presentan un acercamiento a la noción de justicia social desde tal enfoque, para, posteriormente, pensar el asunto del riesgo como derivado de constreñimientos sociopolíticos y económicos, esto es, como expresión de desventajas de los sujetos y de restricciones de las alternativas para elegir seres y haceres valorados, pero también como expresión de las capacidades de los sujetos. Finalmente, se consideran algunas reflexiones acerca del lugar de la salud pública de cara a estas ideas. 


\section{La justicia social en el enfoque de las capacidades}

Siguiendo a Sen ${ }^{14}$, cualquier teoría sobre justicia social debe elegir las características de la realidad en las que ha de centrarse para valorar y juzgar una sociedad como justa o injusta, para evaluar las ventajas generales de un sujeto respecto de otros en un contexto social dado. Así, mientras que algunos teóricos se han centrado en el placer o en la felicidad del individuo -como es el caso del utilitarismo-, o en su ingreso, riqueza o recursos -asuntos privilegiados en ciertos análisis desde la economía-, el enfoque de las capacidades, por su parte, se centra en la libertad como criterio para el juzgamiento de cuán aventajado es un sujeto, en la medida en que posea mayor capacidad u oportunidad real para lograr (ser o hacer) aquello que tiene razones para valorar. Se trata del énfasis en las oportunidades reales para vivir que tienen los sujetos; sin embargo, no es suficiente el disponer de muchas y variadas opciones para elegir, en tanto se trata de que los sujetos estén en condiciones de realizar esas posibilidades de manera autónoma 15.

Desde la perspectiva de Nussbaum 16, el enfoque de las capacidades se erige como una teoría parcial de justicia, en la medida en que busca un mínimo social amplio para todos los sujetos, sobre la base de la idea de igual dignidad humana, para cuya comprensión son centrales la libertad y la habilidad de los sujetos para tomar decisiones que afectan sus vidas. En esta vía, justicia y desarrollo pueden conceptualizarse en términos de las "oportunidades efectivas de las personas para emprender acciones y actividades en las que desean participar y para ser quienes desean ser" 17 (p. 95), por lo cual esta perspectiva hace referencia a la idea de una justicia que no solo reside en poderes gubernamentales o institucionales, sino en las capacidades de los seres humanos, al considerar que una vida es plenamente humana en tanto cuente con un nivel suficiente de oportunidad y actividad para lograr concepciones razonables y diversas sobre una vida buena 18 .

Desde el punto de vista de Sen 14,19, al hablar de capacidades se alude a las posibilidades de los sujetos para lograr funcionamientos (seres y haceres) que les resultan valiosos, por lo cual estas se constituyen en un elemento fundamental de su libertad para conseguir aquella vida que valoran. Así, en el marco del enfoque de las capacidades, la noción de justicia trasciende la lógica de la disponibilidad de recursos o ingresos, y su abordaje implica la consideración de las condiciones sociales y materiales que sustentan las formas de vida de los sujetos. Desde esta perspectiva, la diversidad humana, cuyo lugar es central en sus planteamientos, da lugar a necesidades y satisfactores también diversos, por lo cual los ingresos son un medio para lograr fines (que las personas valoran), más que fines en sí mismos; son valiosos en la medida en que ayudan a los sujetos a lograr vidas decentes. En este sentido, poseer o disponer de un bien no dice nada de lo que los sujetos pueden efectivamente ser y hacer. En consecuencia, el foco es en las libertades sustantivas que las personas poseen para llevar la vida que tienen razones para valorar, en las realizaciones de los sujetos, esto es, en lo que de hecho logran ser o hacer, aunque no se desconoce la necesidad de pensar en los medios que viabilizan tales logros 14,17,19,20,21,22.

En este orden de ideas, un problema central para la teoría de la justicia consiste en la presencia de variaciones entre los sujetos al respecto de la posibilidad de conversión de ingresos, y otros recursos externos, en ventajas personales para lograr aquello que valoran, las cuales son juzgadas desde el punto de vista de la oportunidad 23. La igualdad no significa aquí igualar las capacidades para todos los sujetos; se trata, más bien, de que cada uno pueda avanzar en la expansión de sus posibilidades para conseguir funcionamientos valiosos, toda vez que la justicia no puede ser indiferente a las vidas que los sujetos pueden realmente vivir 14,16.

Ahora bien, el conocimiento científico, por sí mismo, no da cuenta de aquello que es valorado por los sujetos. La comprensión en esta vía requiere basarse en una reflexión ética sobre la dignidad, la libertad y la igualdad de los seres humanos en las circunstancias actuales 18, pero también deriva en la necesidad de ir a la vida de las personas para comprender qué valoran y cuáles son esas valoraciones que subyacen a sus deseos, lo cual implica un alejamiento de las miradas que privilegian únicamente las medias estadísticas, ya que estas no dejan ver la diversidad de asuntos que son relevantes para los sujetos en función del logro de una vida buena 19,20.

Es importante señalar que, desde esta perspectiva, se ha rechazado la idea de la existencia de un modo de vida determinado que pueda ser pensado como universalmente bueno o valioso, esto es, de definir un fundamento absoluto para aquello que constituye una vida buena; en cambio, se ha propuesto que evaluar el modo de vida en que se hallan las personas, implica partir de ellas mismas, de 
sus valores, objetivos, propósitos, de sus fidelidades, obligaciones y su propia concepción del bien 24, ya que, al hacerlo desde un referente externo a sus marcos valorativos, podría estarse imponiendo una idea particular del bien 25 , de un buen desarrollo de la vida 26 . En este contexto, la noción de vida buena, que parte de tradición aristotélica, hace referencia a una vida reflexivamente valorada, esto es, a la vida que las personas tienen razones para valorar 14,24,27. La definición de una vida buena, entonces, no obedece a deseos o gustos personales, sino que es resultado de la capacidad racional de los sujetos 27 , en función de marcos valorativos construidos socialmente.

Desde este enfoque, tal capacidad reflexiva respecto de la valoración de la propia vida, fundamenta la idea del sujeto como agente, capaz de definir metas, objetivos, propósitos, una concepción propia de lo valioso. No obstante, esto no significa un énfasis en la responsabilidad individual (por ejemplo frente al riesgo, vía estilos de vida), en el sentido en que, de acuerdo con Robeyns 17, estos planteamientos no implican un individualismo ontológico, en tanto reconocen las relaciones entre sujetos y su incrustación social, y consideran estas relaciones sociales y las oportunidades para los sujetos, como enmarcadas dentro de estructuras sociales e instituciones que las influencian. En estos términos, el enfoque piensa las condiciones sociales como base para el desarrollo de capacidades.

Siguiendo estas consideraciones y en el marco del propósito de este ensayo, cabe preguntarse si estas valoraciones pueden dar lugar a una asunción de ciertos riesgos al concebirlos como valiosos en el marco de ideas alternativas y plurales de lo que es una vida buena.

\section{Aceptabilidad del riesgo y preferencias adaptativas}

Varios autores se han interesado por pensar en la disposición de los sujetos para tolerar o admitir ciertos riesgos. Por ejemplo, se ha planteado que esta relación con actividades o situaciones potencialmente dañinas puede darse en función de los beneficios percibidos por los sujetos respecto de su exposición a los riesgos que conllevan 2,28. También se ha afirmado que la aceptabilidad del riesgo es una construcción social, asociada a valores contextuales particulares y a una idea de equidad y de justicia (frente a la distribución del riesgo mismo) de las poblaciones implicadas en tales situaciones o actividades riesgosas 12 .

De acuerdo con Spink 13, cuya postura no desconoce que las posiciones sobre el futuro (y la idea de riesgo emerge como posibilidad de hablar de eventos futuros) están asociadas a condiciones materiales de existencia de los sujetos, en la sociedad actual y en el marco de nuevas sensibilidades frente al riesgo, nuevas formas de interpretarlo y nuevas funciones de esas interpretaciones en la vida social, los sujetos pueden elegir asumir el riesgo, por lo cual este ya no es vivenciado como una amenaza susceptible de ser cuantificada, controlada y objeto de medidas preventivas, sino en términos de novedad, imponderabilidad y aventura 29 . Frente a esta postura, se ha señalado que implica reexaminar la forma como la salud pública ha concebido tradicionalmente el riesgo (a partir de modelos estadísticos), la cual ha excluido dimensiones del riesgo que no necesariamente llevan el sello de lo indeseado, que no conceptualizan el riesgo como algo que puede ser procurado deliberadamente y cuya comprensión podría incorporar el planteamiento canguilhemiano de la salud como la posibilidad de abusar de la salud misma ${ }^{30}$. No obstante, existe la necesidad de diferenciar entre aquellos riesgos que son socialmente legitimados y otros que la salud pública ha considerado históricamente como inaceptables; en consecuencia, la positividad del riesgo estaría limitada a aquellos riesgos médicamente correctos o tolerables 31 .

Otros autores han afirmado que la aceptabilidad del riesgo no siempre responde a una elección del sujeto de entre una gama de alternativas. En este sentido, hay grupos poblacionales (como los jóvenes, los trabajadores informales, las mujeres en ciertas condiciones societales, y la lista podría ampliarse de manera exponencial) que precisan tomar riesgos en función de implicarse y hacer uso de beneficios de la sociedad de consumo, como forma de sobrevivir o tener reconocimiento, y no solo por las emociones extremas que pueda procurar la exposición a amenazas o por una búsqueda de placer o de sentido de vida. En estos casos, aunque el riesgo asume el carácter epidemiológico de probabilidad de los sujetos de padecer daños en su salud dada la exposición a factores peligrosos determinados, tal exposición es independiente de la voluntad de los sujetos para asumirlos, ya que se trata de un riesgo derivado de sus condiciones particulares de vida, de sus situaciones de vulnerabilidad, asociadas a la 
realidad material de millones de personas en el mundo que parecen vivir en peligro constante 9,10,11, en el marco de diferencias injustas que imponen formas de estar en el mundo y que han sido reconocidas y abordadas, en distintos niveles, a partir de diversos puntos de vista en el ámbito de la salud pública, tanto desde posturas asociadas a la idea de determinantes sociales de la salud 32,33, como desde la propuesta de la determinación social de la salud, propia de lecturas desde la epidemiología crítica y la medicina social latinoamericana 34,35 .

Estos asuntos llevan a considerar la necesaria relación entre riesgo y justicia social, pues ¿qué tanto el riesgo que se acepta, que se admite o integra en la cotidianidad de los sujetos, responde a una elección por este a partir de rasgos, aptitudes y/o juicios acerca de las situaciones (o actividades) amenazantes como valiosas para sus vidas?, ¿qué tanto los sujetos tienen oportunidades reales para realizar y ser aquello que valoran?, ¿elegirían ellos asumir los riesgos que asumen si tuvieran otras posibilidades y condiciones de vida?

Estos interrogantes hacen pensar otra vía para leer esas formas de asunción e incorporación del riesgo en la cotidianidad de sujetos y grupos. Desde la perspectiva del enfoque de las capacidades, una persona puede sentirse feliz, satisfecha, sana, como una estrategia adaptativa aún en graves circunstancias de vida o, en otras palabras, un sujeto que carece de oportunidades puede terminar ajustando sus preferencias o actitudes para lograr un sentido de satisfacción relativo 36. Podría ser el caso, por ejemplo, de un sujeto que realiza un oficio peligroso (como la minería de hecho) en un contexto desfavorable para su salud, oficio en el cual puede sentirse conforme y complacido porque este posibilita su subsistencia; sin embargo, algunas prácticas de este tipo son llevadas a cabo en entornos en los que no hay otras opciones para el sustento, o no se cuenta, dentro de la actividad misma, con alternativas más seguras para su realización.

En este orden de ideas, se trata de la posibilidad (y no necesariamente de la elección) de vivir en la precariedad y de sentirse capaz de "vivir bien" en tales condiciones; asunto este que, desde algunas perspectivas, puede pensarse como con una cierta positividad, en la medida en que permite a los sujetos llevar una vida con algún nivel de bienestar aún en tales circunstancias. Así, desde discursos (debatidos) como el de la resiliencia, estas situaciones se constituyen en "deseables", en una "respuesta lógica" ante las crisis y la naturalización de las inseguridades, bajo el imperativo para los sujetos de demostrar sus habilidades para vivir con el peligro 4; esto en el marco de sociedades que valorizan al individuo, pero que también promueven su vulnerabilidad 3 , que no buscan resolver precariedades ni amenazas para salud y la vida, sino hacer que los sujetos renuncien a la idea y a la posibilidad de seguridad, en la medida en que acogen la necesidad de exponerse a riesgos como un medio para estar bien 4 .

En virtud de ello, centrar la mirada solo en cómo las personas se sienten acerca de sus vidas, puede dar lugar a centrarse en una falsa consciencia y devaluar significados y entendimientos que forman la base de decisiones y acciones de los sujetos; desde este punto de vista, lo relevante es la calidad de vida que los sujetos viven, definida también por las condiciones objetivas que la soportan 14,37. No se trata de un rechazo a la idea del bienestar como relevante para el análisis de las formas de vida de los sujetos, sino al hecho de concebirlo como la única variable importante en este análisis, así también, a pensarlo como exclusivamente un estado mental, como ligado únicamente al ingreso, o como asociado al principio utilitario de la satisfacción de deseos 17,24.

En consecuencia, no ha de inferirse el valor de una capacidad únicamente a partir de preferencias de los sujetos, las cuales pueden estar distorsionadas por su inmersión en condiciones de vida desfavorables normalizadas 16 , con lo cual se alude a la posibilidad de que las personas elijan bajo techos limitados de elección; es decir, en el marco de una limitación de opciones, sus decisiones pueden estar constreñidas y derivar en una pérdida de la capacidad para tener aspiraciones diferentes a las alternativas ofrecidas por el medio. Estos planteamientos implican la consideración de si se trata de un asunto auténtico de valoración (por ejemplo de una situación riesgosa) o si las personas prefieren aquello que prefieren porque no tienen otras opciones, por lo que la pregunta no es solo por lo que los sujetos eligen, sino también por las razones por las cuales valoran lo que valoran. En este sentido, es tan importante lo elegido como el proceso mismo de elección y el conjunto de funcionamientos alternativos frente a los cuales el sujeto hizo ejercicio de su libertad 14.

Como forma de ilustrar estos planteamientos, es posible reflexionar sobre algunos escenarios en los cuales las personas "viven con el riesgo", por ejemplo con las amenazas propias de su actividad laboral o las derivadas de contextos de precariedad en diversos ámbitos (social, económico, político, 
sanitario). Actualmente, con base en los presupuestos del modelo socioeconómico imperante, se ha promovido una apuesta extractivista en el mundo, contexto en el cual actividades como la minería han tenido un impulso relevante; es el caso colombiano (así como de otros países latinoamericanos), donde, a partir de su promoción como país minero y desde instancias gubernamentales, se ha buscado una mayor inversión extranjera a través de la incursión de empresas multinacionales en su jurisdicción, sin las necesarias regulaciones en materia social y ambiental. Este asunto ha sido analizado y objeto de importantes críticas desde perspectivas de justicia, pues las poblaciones de cuyos territorios se arrancan los recursos naturales, no reciben beneficios asociados a la explotación y sí, en cambio, los impactos negativos derivados de esta práctica 38,39,40.

En este escenario, las poblaciones pueden dejar sus trabajos tradicionales para dedicarse a labores mineras llevadas a cabo por las grandes compañías extractivistas, bajo presupuestos de mayor seguridad industrial, cobertura en servicios de salud, estabilidad salarial, beneficios para las familias. No obstante, si estas personas contaran con la garantía de sus derechos al trabajo, a la salud, a la educación, ¿necesariamente elegirían cambiar sus formas tradicionales de vida? Las personas que residen en las zonas de influencia de la actividad también podrían decidir seguir viviendo allí, aún cuando esta circunstancia represente riesgos para su salud, pero, ¿continuarían en el lugar si tuvieran otras opciones de vida?, ¿podrían ellas participar de las decisiones frente a la incursión o no de estas industrias en sus territorios? A partir de reflexiones como estas, desde el punto de vista del enfoque de las capacidades, escoger de entre un conjunto de funcionamientos aquellos que se conciben como valiosos, requiere de un acto de elección que puede estar influenciado o incluso constreñido por estructuras sociales 17 .

Ahora bien, para el caso de una práctica como la minería tradicional, a la cual, desde perspectivas expertas técnico-científicas se han asociado diversos riesgos, cabe considerar algunas posibilidades. Puede ser que las personas hayan deseado hacer de la minería su actividad (aún con los riesgos que supone) teniendo en cuenta, por ejemplo, aspectos asociados a la tradición o a una forma particular de relación con la tierra en busca de sustento. No obstante, puede ser también que no hayan encontrado otras posibilidades laborales, por lo cual la minería podría haberse constituido en la opción más viable para el sustento. Si este es el caso, el constreñimiento de la libertad para elegir la vía laboral privilegiada puede ser pensado como una violación del "aspecto de proceso" de la libertad (del poder decidir por sí mismo qué hacer), aún en los casos en los cuales las personas, en otras circunstancias, hubieran optado por elegir libremente esta actividad.

Asimismo, se ve afectado el "aspecto de oportunidad" de la libertad, en el sentido en que las personas no contarían con una relación de oportunidades que incluyan variedad de opciones, asunto que podría verse más quebrantado en los casos en los que no solo se vieran obligadas a introducirse en la actividad minera dadas sus condiciones particulares de vida (lo que las forzaría a realizar una labor no necesariamente elegida por ellas), sino también cuando se vieran forzadas a dedicarse a una práctica que de otra manera (bajo otras circunstancias) no elegirían. Así pues, podría pensarse que algunas personas manifiestan su inclinación por esta labor, sin la consciencia de que esta preferencia está constreñida por la falta de otras oportunidades ofrecidas por el entorno en el que ocurren sus vidas. En estos casos, la aceptación de la actividad y de los riesgos inherentes a ella puede concebirse como expresión de una limitación para el ejercicio de la libertad, y, consecuentemente, como una restricción de las capacidades de los sujetos implicados.

\section{Limitación de opciones para elegir y exposición a riesgos: de la naturalización a la agencia}

Estas ideas, que permiten pensar en una naturalización de la imposibilidad para elegir entre una variedad de alternativas, implican que el rango de oportunidad puede ser estrecho, no porque los sujetos opten por ello en correspondencia con sus talentos, habilidades o valoraciones, sino porque las opciones limitadas pueden ser lo más usual en una sociedad; así, algunos estados de cosas o ciertos funcionamientos, pueden ser los más comúnmente logrados en contextos particulares. En estos escenarios, la idea de igualdad de oportunidades (que pueden ser restringidas para todos), solo tiene sentido cuando tales oportunidades resultan valiosas para los sujetos 18 , sobre la base de la idea de libertad efectiva como un fin. En este orden de ideas, no se trata únicamente de reflexionar en torno 
a las opciones con las cuales cuentan los sujetos, sino también alrededor del proceso de la libertad, es decir, sobre la pregunta por las razones por las cuales los sujetos eligen aquello que eligen, si sus elecciones responden a sus concepciones de lo bueno para sus vidas 14 , valoraciones que, además, son producto de construcciones desde los significados compartidos con otros, que no emergen de manera natural, sino a partir de la interacción social 37.

Así, no basta solo con la pregunta por el bienestar de los sujetos, sino por las oportunidades (o libertades sustanciales) disponibles para cada uno de ellos, las cuales se constituyen, de acuerdo con Nussbaum 16, en el bien crucial que las sociedades deberían promover. Desde esta lógica se reconoce la construcción social de significados sobre el bienestar, la calidad de vida, lo riesgoso para la salud y para la vida, nociones que son instituidas en contextos socioculturales específicos, lo cual deriva no solo en la necesaria consideración de estructuras sociales e instituciones que posibilitan o dificultan a los sujetos perseguir libertades en relación con otros en la sociedad, sino que se trata también de un enfoque comprometido con la autodefinición y la agencia de los sujetos, y pluralista en términos de valores 14,24 .

En este sentido, como se ha mencionado, trabajar puede ser visto como un funcionamiento valioso, pero es preciso preguntarse por trabajar en qué, para qué, bajo cuáles circunstancias. Asimismo, puede considerarse la asunción de un riesgo como algo valorado positivamente por los sujetos, pero sería necesario indagar por los motivos de la aceptación de ese riesgo y por las alternativas diversas de entre las cuales un sujeto eligió exponerse a él, aún cuando puede representar daños potenciales para su salud o para su vida. Estas cuestiones remiten al terreno de las capacidades, de la libertad de elección de (o para lograr) funcionamientos considerados valiosos en correspondencia con diversas ideas del buen vivir, del trabajar y del incorporar riesgos en la cotidianidad de una vida que le resulte significativa al sujeto del que se trate.

Estos planteamientos llevan al ámbito de la agencia, la cual hace referencia a la capacidad de acción del sujeto para elegir la vida que tiene razones para valorar. En estos términos, los seres humanos son vistos como participantes activos (si se les da la oportunidad) en la configuración de su destino, y no como receptores pasivos de condicionamientos 27 . Siguiendo a Restrepo 41, la idea del ser humano como agente está fundamentada en la capacidad reflexiva de los sujetos en torno a la valoración de la propia vida. En esta línea de ideas, la noción de "libertad de agencia", relevante desde el enfoque de las capacidades, alude a la libertad del sujeto para buscar los propósitos que considera valiosos a partir de su concepción del bien (que construye en interacción con otros), aún en los casos en los que tales propósitos vayan en oposición a su propio bienestar 14,24,37.

Consecuentemente, la aceptación de ciertos riesgos o su incorporación en la cotidianidad de los sujetos, puede ser pensada como la expresión misma de su libertad de agencia, así como de su libertad de bienestar ${ }^{37}$. No obstante, este planteamiento supone el análisis de las situaciones de aceptación del riesgo a la luz de qué tanto eso (riesgoso) que se elige, se corresponde con una concepción de vida buena, asociada a una idea de realización de sí, la cual implica un propósito y marcos valorativos propios 25,41 . Esta posibilidad de concebir una noción propia de vida buena por parte de los sujetos, entraña también un desarrollo pleno de su capacidad de agencia, así como la posibilidad de razonamiento y de reflexión sobre la vida (proceso donde el sujeto ejerce su capacidad de juicio) 14 , en la medida en que, en estos casos, los sujetos tendrían razones para valorar el exponerse a ciertos riesgos, incluso, posiblemente, en función de evitar otros riesgos percibidos como mayores, ya que al hacer juicios personales, cada sujeto selecciona ponderadores de situaciones y funcionamientos, de acuerdo con la forma en que crea que es razonable 23.

En síntesis, elegir riesgos puede ser expresión de limitaciones de las capacidades, por cuanto el asunto de una supuesta "voluntariedad" para exponerse a estos tendría que ser considerada en los análisis. Por otro lado, la opción por los riesgos puede constituirse en una expresión de capacidad, en el sentido en que elegir algo riesgoso puede ser visto como una realización valiosa y derivar de una distinción sensata entre optar por algo potencialmente dañino cuando el sujeto cuenta con otras opciones por las cuales podría decidirse 22. Así, la opción por el riesgo puede responder o no a una concepción de vida buena.

Estos planteamientos derivan en una necesaria revisión de posturas que juzgan a ciertos sujetos como socialmente vulnerables, las cuales se tornan en una esencialización de aquellos a quienes incluyen en tal categoría. Entender el riesgo y su aceptación como posible expresión de las capacidades y 
no solo (y en todos los casos) como una restricción de las mismas, comporta comprender un sujeto con potencia para desarrollar agencia (capacidad de ser agente), con la posibilidad de definir su propia concepción de lo bueno y de lo valioso, y de dirigir su vida en consecuencia.

\section{Reflexiones para la salud pública}

Para concluir, cabe plantear la necesidad de una reflexión en esta materia desde la salud pública, pues si las personas eligen el riesgo, bien por constreñimiento en sus elecciones o bien como ejercicio de su libertad a partir de una idea razonada de vida buena, existen retos importantes para este campo de conocimiento y de acción, en la búsqueda de una vida mejor para las poblaciones. Este asunto lleva a pensar la pertinencia de adelantar análisis y acciones para identificar situaciones y valoraciones que subyacen a la forma como los sujetos se relacionan con aquello leído como riesgoso, lo cual implica situarse en los contextos particulares de estudio para entender el riesgo y las relaciones plurales que los sujetos establecen con él. No obstante, esta contextualización del riesgo, lejos de pensarse como exclusivamente centrada en individuos, involucra también un abordaje de las condiciones sociales y materiales que, en la trayectoria de los sujetos, soportan o no la posibilidad de vivir una vida reflexivamente valorada 18 , dado el interés del enfoque de las capacidades por analizar la influencia que, sobre los sujetos, tienen los ordenamientos sociales 17.

En estos términos, el asunto de la admisión del riesgo, leído a la luz del enfoque de las capacidades, supone preguntas que precisan ser consideradas en las evaluaciones del riesgo, en función de ampliar su alcance más allá de la tradicional concepción probabilística: ¿por qué los sujetos aceptan ciertos riesgos?, ¿qué motiva esa aceptación o incorporación de tales riesgos en su cotidianidad?, ¿esta aceptación responde realmente a una elección de entre una gama de alternativas? Finalmente, ¿cuál es la lectura de estos asuntos desde la salud pública? En esta vía, cabe reflexionar acerca de si se trata de una comprensión de las amenazas para la salud y para la vida como asociadas principalmente a constreñimientos por limitación de opciones, lo cual puede responder a lecturas desde posturas centradas en los procesos sociales que configuran resultados en salud; o si se trata de pensar la asunción de riesgos solo como una elección del sujeto desde sus preferencias, con fundamento en nociones como las de estilos de vida y factores de riesgo, o en la idea de que los sujetos, bien informados, pueden tomar "decisiones adecuadas" para prevenir riesgos en salud.

Un abordaje bajo la perspectiva del enfoque de las capacidades, podría dar lugar a pensar que ambas posturas, entendidas como únicas y excluyentes, son insuficientes para explicar las relaciones de los sujetos con el riesgo, lo cual supondría, para la salud pública, el no desconocer situaciones sociales que limitan la elección de aquello que los sujetos tienen razones para valorar (y que pueden ser naturalizadas), como tampoco la posibilidad de que ellos opten por asuntos que incluso vayan en contravía de la idea normalizada de bienestar. 


\section{Agradecimientos}

La autora agradece al profesor Diego Alveiro Restrepo Ochoa, miembro del comité de dirección de su tesis doctoral en la Facultad Nacional de Salud Pública, Universidad de Antioquia, por la lectura y sugerencias aportadas para enriquecer este ensayo. Igualmente, a COLCIENCIAS, ente financiador de su proceso de formación doctoral (Convocatoria 647 de 2014).

\section{Referencias}

1. Castiel LD, Rodrigues GM, Santos FM. Corriendo el riesgo. Una introducción a los riesgos en salud. Bogotá: Universidad del Rosario/ Rio de Janeiro: Editora Fiocruz; 2015.

2. Slovic P. The perception of risk. Washington DC: Earthscan; 2000.

3. Castel R. La inseguridad social. ¿Qué es estar protegido? Buenos Aires: Manantial; 2013.

4. Evans B, Reid J. Una vida en resiliencia: el arte de vivir en peligro. México DF: Fondo de Cultura Económica; 2016.

5. Almeida Filho N, Castiel LD, Ayres JR. Riesgo: concepto básico de la epidemiología. Salud Colect 2009; 5:323-44.

6. Ayres JR. Acerca del riesgo. Para comprender la Epidemiología. Buenos Aires: Lugar Editorial; 2005.

7. Almeida Filho N. La ciencia tímida. Ensayos de deconstrucción de la epidemiología. Buenos Aires: Lugar Editorial; 2000.

8. Hayes M. On the epistemology of risk: language, logic and social science. Soc Sci Med 1992; 35:401-7.

9. Ayres JR, Paiva V, França Jr. I. Conceitos e práticas de prevenção: da história natural da doença ao quadro de vulnerabilidade e direitos humanos. In: Paiva V, Ayres JR, Buchalla C, editores. Vulnerabilidade e direitos humanos: prevenção e promoção da saúde. Livro I - da doença à cidadania. Curitiba: Juruá Editora; 2012. p. 71-94.

10. Weller SI. Debate sobre o artigo de Mary Jane P. Spink. Cad Saúde Pública 2001; 17:1300-1.

11. Souza ER. Quando viver é o grande risco-aventura. Cad Saúde Pública 2001; 17:1291-2.

12. Douglas M. La aceptabilidad del riesgo según las ciencias sociales. Barcelona: Paidós; 1996.

13. Spink MJ. Trópicos do discurso sobre risco: risco-aventura como metáfora na modernidade tardia. Cad Saúde Pública 2001;17:1277-88.

14. Sen A. La idea de la justicia. Ciudad de México: Editorial Taurus; 2009.

15. Saldarriaga MA. El sujeto activo: antropología política en Amartya Sen. Eidos 2010; (13):5475.

16. Nussbaum M. Crear capacidades: propuesta para el desarrollo humano. Barcelona: Paidós; 2012.

17. Robeyns I. The capability approach: a theoretical survey. J Hum Dev 2005; 6:93-114.

18. Venkatapuram S. Health justice: an argument from the capabilities approach. Cambridge: Polity Press; 2011. 277 p.

19. Sen A. Capacidad y bienestar. In: Nussbaum M, Sen A, editores. La calidad de vida. Tlalpan: Fondo de Cultura Económica; 1996. p. 54-83.

20. Robeyns I. The capability approach in practice. J Polit Philos 2006; 14:351-76. 
21. Comim F. Operationalizing Sen's capability approach. In: Conference Justice and Poverty: Examining Sen's Capability Approach. Cambridge: University of Cambridge; 2001. p. 1-16.

22. Sen A. Pobre, en términos relativos. Comercio Exterior 2003; 53:413-6.

23. Sen A, Foster J. Espacio, capacidad y desigualdad. Comercio Exterior 2003; 53:416-7.

24. Sen A. Bienestar, justicia y mercado. Barcelona: Paidós; 1997.

25. Restrepo OD. Los jóvenes y la vida buena: aportes del enfoque de las capacidades para el abordaje de la salud mental en el campo de la salud pública [Tesis de Doctorado]. Medellín: Universidad de Antioquia; 2015.

26. Ziegler H. Subjective well-being and capabilities: views on the well being of youth persons In: Andresen S, Diehm I, Sander U, Ziegler H, editores. Children and the good life: new challenges for research on children. London/New York: Springer; 2010. p. 91-102.

27. Sen A. Desarrollo y libertad. Buenos Aires: Planeta; 2000

28. Slovic P, Weber EU. Percepción del riesgo generado por eventos extremos. In: Estrategias de Manejo de Riesgo en un Mundo Incierto. New York: Center fosr Decision Sciences, Columbia University; 2002.

29. Spink MJ, Pereira AB, Burin LB, Silva MA, Diodato PR. Usos do glossário do risco em revistas: contrastando "tempo" e "públicos". Psicol Reflex Crít 2008; 21:1-10.

30. Canguilhem G. Lo normal y lo patológico. Buenos Aires: Siglo XXI; 1971.

31. Caponi S. La aceptabilidad del riesgo. Cad Saúde Pública 2001; 17:1306-7.

32. Marmot M, Wilkinson R. Social determinants of health. Oxford: Oxford University Press; 2006.
33. Organización Mundial de la Salud. Cerrando la brecha: la política de acción sobre los determinantes sociales de la salud. Documento de Trabajo. Río de Janeiro: Organización Mundial de la Salud; 2011.

34. Breilh J. Las tres 'S' de la determinación de la vida: 10 tesis hacia una visión crítica de la determinación social de la vida y la salud. In: Passos Nogueira R, editor. Determinação social da saúde e reforma sanitária. Río de Janeiro: Centro Brasileiro de Estudos de Saúde; 2010. p. $87-$ 125. (Coleção Pensar em Saúde).

35. Breilh J. La determinación social en la salud. Una perspectiva emancipadora de la investigación y acción, basada en la determinación social de la salud. In: Taller Latinoamericano sobre Determinantes Sociales de la Salud. Ciudad de México: Asociación Latinoamericana de Medicina Social; 2008. p. 14-29.

36. Spring J. Rights and well-being in Amartya Sen's value theory. J Value Inq 2011; 45:13-26.

37. Deneulin S, McGregor J. The capability approach and the politics of a social conception of wellbeing. Eur J Soc Theory 2010; 13:501-19.

38. Infante C. Pasivos ambientales mineros: barriendo bajo la alfombra. Lima: Observatorio Conflictos Mineros América Latina; 2011.

39. Gudynas E. El nuevo extractivismo progresista. Revista Nueva Crónica 2010; (55):7.

40. Gudynas E. El nuevo extractivismo progresista. Tesis sobre un viejo problema bajo nuevas expresiones. In: Acosta A, Gudynas E, Houtart F, Ramírez H, Martínez J, Macas L, editores. Colonialismos del siglo XXI. Negocios extractivos y defensa del territorio en América. Barcelona: Icaria; 2011. p. 75-92.

41. Restrepo OD. La salud y la vida buena: aportes del enfoque de las capacidades de Amartya Sen para el razonamiento ético en salud pública. Cad Saúde Pública 2013; 29:2371-82. 


\section{Abstract}

This article discusses the notions of risk and risk acceptability from a social justice perspective, especially in light of the capability approach proposed by Amartya Sen. The article argues that risk can be the expression of restrictions on subjects' $c a-$ pabilities, deriving from social disadvantages that can be taken for granted in their daily realities. On the other hand, risk can be viewed as an expression of capability in cases where subjects have accepted or admitted the risk through the exercise of freedom, as long as the subjects that relate to the risk do so in keeping with their idea of a good life, the building of which implies the full development of capability for agency. The article concludes with some thoughts on the issues of risk and risk acceptability in the sphere of public health.

Risk-taking; Risk; Social Justice; Freedom

\section{Resumo}

O artigo aborda as noções de risco e aceitação do risco desde uma perspectiva da justiça social, especialmente, através do enfoque de capacidades proposto por Amartya Sen. É argumentado que o risco pode ser a expressão de restrições das capacidades dos sujeitos, partindo de desvantagens sociais que podem se encontrar normalizadas no seu cotidiano. Por outro lado, pode ser interpretado como expressão de capacidade, naqueles casos nos quais é aceito ou admitido através do exercício da liberdade, sempre que os sujeitos que assim se relacionam com o arriscado, concebam isso em sintonia com sua ideia de uma vida boa, cuja construção implica o desenvolvimento pleno da capacidade de agência. Finalmente, se apresentam algumas reflexões sobre a matéria dentro do âmbito da saúde pública.

Assunção de Riscos; Risco; Justiça Social; Liberdade
Recibido el 02/Oct/2017

Versión final presentada el 29/Dic/2017

Aprobado el 02/Feb/2018 